\title{
$\Delta I=\frac{1}{2}$ rule in the light of two-dimensional QCD
}

\author{
John Ellis* \\ Theoretical Physics Division, CERN, CH-1211 Geneva 23, Switzerland \\ Yitzhak Frishman ${ }^{\dagger}$ \\ Department of Particle Physics, Weizmann Institute of Science, Rehovot 76100, Israel \\ Amihay Hanany \\ IAS, Princeton, New Jersey 08540 \\ Marek Karliner $\$$ \\ School of Physics and Astronomy, Raymond and Beverly Sackler Faculty of Exact Sciences, Tel-Aviv University, 69978 Tel-Aviv, Israel
}

(Received 2 December 1996)

\begin{abstract}
We calculate in two-dimensional $\mathrm{QCD}\left(\mathrm{QCD}_{2}\right)$ the ratios of baryonic matrix elements of $\Delta I=2$ and $\Delta I=0$ four-fermion operators, with a view to understanding better the mechanism of $\Delta I=1 / 2$ enhancement in $\mathrm{QCD}_{4}$. We find relatively small suppressions of both the scalar-scalar and vector-vector $\Delta I=2$ four-fermion operators. We discuss the possible implications of these results, in view of a suggestion that gluon condensation may be an important contributing factor in the $\Delta I=1 / 2$ enhancement seen in $\mathrm{QCD}_{4}$. At the technical level, our calculation of the vector-vector operator matrix element requires a treatment of the time dependence of the $\mathrm{QCD}_{2}$ soliton which had not been developed in previous phenomenological calculations within this model. [S0556-2821(97)01807-9]
\end{abstract}

PACS number(s): 12.38.Aw, 11.10.Kk, 12.38.Lg

\section{INTRODUCTION}

Two-dimensional QCD $\left(\mathrm{QCD}_{2}\right)$ is often a useful testing ground for ideas concerning nonperturbative effects in QCD in four dimensions $\left(\mathrm{QCD}_{4}\right)$. Among the issues studied in $\mathrm{QCD}_{2}$ have been those associated with confinement [1], the appearance of baryons $(B)$ as solitons [2], the existence of constituent quarks and their relation to current quarks [3], and quark-antiquark condensates [4]. However, care must be exercised in carrying conclusions over directly from $\mathrm{QCD}_{2}$ to $\mathrm{QCD}_{4}$, since there are essential differences between the dynamics in the different dimensions. Among these differences are the dimensionless nature of the $\mathrm{QCD}_{4}$ gauge coupling as opposed to the dimensional nature of the $\mathrm{QCD}_{2}$ gauge coupling, the associated differences in the infrared behaviors of $\mathrm{QCD}_{2}$ and $\mathrm{QCD}_{4}$ (at least in perturbation theory), and the absence in $\mathrm{QCD}_{2}$ of spontaneous chiral symmetry breaking and the pseudo-Goldstone boson interaction of the light pions in $\mathrm{QCD}_{4}$. Another important difference, to which we shall return in this paper, is the fact that gluons in $\mathrm{QCD}_{2}$ do not have any physical polarization states. Thus in two dimensions (2D) the analogue of the glue condensate in the vacuum $\left\langle 0\left|F^{2}\right| 0\right\rangle$ may only come from mixing with quark-antiquark pairs, and hence is expected to be much smaller than in $\mathrm{QCD}_{4}$. In particular, it vanishes in the large$N_{c}$ limit.

$\mathrm{QCD}_{2}$ has been used previously to calculate certain non-

\footnotetext{
*Electronic address: john.ellis@ @ern.ch

${ }^{\dagger}$ Electronic address: fnfrishm@wicc.weizmann.ac.il

¥Electronic address: hanany@sns.ias.edu

${ }^{\S}$ Electronic address: marek@vm.tau.ac.il
}

perturbative matrix elements of local operators, such as quark-antiquark condensates in baryons $\langle B|\bar{q} q| B\rangle$ [4], with results ${ }^{1}$ that compare well with Skyrme models [5] and phenomenological determinations in $\mathrm{QCD}_{4}$ [6], and may cast light on observations of apparent violations of the OkuboZweig-Iizuka (OZI) rule in meson-baryon couplings [7]. Nonleptonic weak decays are controlled by the matrix elements of higher-dimensional operators, such as $\langle B|(\bar{q} q)(\bar{q} q)| B\rangle$, in $\mathrm{QCD}_{4}$. These matrix elements exhibit some puzzling features, in particular large enhancements of $\Delta I=\frac{1}{2}$ transitions compared with $\Delta I=\frac{3}{2}$ transitions (for a review, see, for example, [8]). It seems that calculable perturbative $\mathrm{QCD}_{4}$ renormalization effects below the weak scale [9] cannot explain all the large $\Delta I=\frac{1}{2}$ enhancement observed, and hence that the major part of it must be nonperturbative in nature. The main purpose of this paper is to calculate an analogue in $\mathrm{QCD}_{2}$ of the enhancement of the $\Delta I=\frac{1}{2}$ component of nonleptonic decay amplitudes, ${ }^{2}$ in the hope of casting light on the nature of the nonperturbative enhancement mechanism active in $\mathrm{QCD}_{4}$.

This calculation requires certain improvements in the technology developed previously for calculations in $\mathrm{QCD}_{2}$ [2]. As is well known, baryons in QCD may be described as solitons in a bosonized formulation. The collective coordinates of the soliton must be quantized consistently, introducing a time dependence into the soliton wave function which

\footnotetext{
${ }^{1}$ Of particular interest have been ratios of quark condensates, such as the ratio of the strange quark condensate to the sum of all condensates, for which a smooth nonzero limit exists in the limit of vanishing quark masses.

${ }^{2}$ For one previous approach to this problem using $\mathrm{QCD}_{2}$, see [10].
} 
corresponds to the angular momentum of the baryonic soliton (Skyrmion) in $\mathrm{QCD}_{4}$ [11]. Previous calculations of baryonic matrix elements of simple local operators such as $\bar{q} q$ [4] did not require an explicit treatment of this collectivecoordinate quantization. However, this is necessary for the calculation of the baryonic matrix element of the nonleptonic weak Hamiltonian, which contains current-current terms $J^{\mu} J_{\mu}$, since the time component $J^{0}$ involves time derivatives of the bosonic fields in the soliton.

Our main physical result is a relatively small numerical factor between the nonperturbative $\Delta I=\frac{1}{2}$ and $\Delta I=\frac{3}{2}$ matrix elements, which appears inadequate to explain the $\Delta I=\frac{1}{2}$ enhancement observed in $\mathrm{QCD}_{4}$, even allowing for the perturbative enhancement factors calculated in $\mathrm{QCD}_{4}$ [9]. We interpret this result as indicating that the observed $\Delta I=\frac{1}{2}$ enhancement in $\mathrm{QCD}_{4}$ may be due to particular features of the nonperturbative $\mathrm{QCD}_{4}$ dynamics that are absent in $\mathrm{QCD}_{2}$. A candidate for this is the gluon condensate, which has indeed been proposed [12] as the main mechanism responsible for the observed $\Delta I=\frac{1}{2}$ enhancement in $\mathrm{QCD}_{4}$, and which is relatively small in two dimensions, even vanishing in the large- $N_{c}$ limit.

It is interesting to compare this result to previous calculations in four-dimensional Skyrme models [13], which found a magnitude of $\Delta I=1 / 2$ enhancement that is sensitive to the model-dependent spatial wave function. In our twodimensional case, the integral over this wave function can be carried out analytically and exactly. The Skyrme models incorporate correctly the physics of quark condensation and chiral symmetry breaking in four dimensions, but do not in general include representations of the gluonic degrees of freedom in $\mathrm{QCD}_{4}$, and in particular the physics of gluon condensation.

\section{COMPUTATION OF THE MATRIX ELEMENTS FOR THE QUADRILINEAR SCALAR INTERACTION}

As a warmup exercise, we start in this section by calculating an analogue of the $\Delta I=1 / 2$ enhancement in the ratio between matrix elements of four-fermion scalar densities in $\mathrm{QCD}_{2}$. Our eventual aim is to calculate the analogous ratio between matrix elements of products of Lorentz-vector currents, as this is more comparable to the quantity of interest in four dimensions. However, the latter calculation involves more technical aspects of baryonic solitons in $\mathrm{QCD}_{2}$, so to get the flavor of the physics of the computation we first tackle the simpler case of scalar densities with $I_{3}=0$ and $I=2,0$, which we denote by $T_{20}$ and $T_{00}$, respectively.

To calculate the matrix elements of these operators, we first review the static classical soliton which describes a baryon in $\mathrm{QCD}_{2}$ [2]. In the strong-coupling limit this is given by a solution to a sine-Gordon equation, as seen in Eq. (5.4) of Ref. [2], namely

$$
\varphi(x)=\frac{4}{\beta} \arctan [\exp (\beta \sqrt{2} m x)],
$$

where $\beta=\sqrt{4 \pi / N_{c}}$ is the coupling constant of the sineGordon theory, $m / \beta$ is the mass of the soliton, and $m$ is related to the common bare mass of the quarks by a renor- malization group relation appropriate to two dimensions. The semiclassical quantization of this soliton entails the introduction of time-dependent coordinates $z_{i}, i=1, \ldots, N_{f}$ [2]. In terms of these coordinates, the relations between $\overline{q q}$ bilinear densities and the scalar fields follow from the standard bosonization correspondence:

$$
\overline{q_{i}} q_{i}=\left\{1+(\cos \beta \varphi-1)\left|z_{i}\right|^{2}\right\} \Lambda,
$$

where $\Lambda$ is an appropriate mass scale [4].

On physical grounds, and in order to calculate finite terms in the classical expression for the four-fermion operators, we need to subtract the vacuum expectation values of the operators. This leads to the relations

$$
\begin{aligned}
\bar{q}_{i} q_{i} & \overline{q_{j}} q_{j}-\left\langle 0\left|\overline{q_{i}} q_{i} \overline{q_{j}} q_{j}\right| 0\right\rangle \\
& =\left\{\left[1+(\cos \beta \varphi-1)\left|z_{i}\right|^{2}\right]\left[1+(\cos \beta \varphi-1)\left|z_{j}\right|^{2}\right]-1\right\} \Lambda^{2} \\
& =\left\{(\cos \beta \varphi-1)\left(\left|z_{i}\right|^{2}+\left|z_{j}\right|^{2}\right)+(\cos \beta \varphi-1)^{2}\left|z_{i}\right|^{2}\left|z_{j}\right|^{2}\right\} \Lambda^{2},
\end{aligned}
$$

where the term proportional to -1 at the end of first row comes from the vacuum subtraction.

Next we turn to the computation of the expectation values (3). In the semiclassical approximation, we may separate out the classical contribution, which amounts to an integral over the spatial coordinate $x$. Setting $\beta \sqrt{2} m=\mu$, and using some elementary $x$ integrals listed in the Appendix, we find

$$
\left\langle\overline{q_{i}} q_{i} \bar{q}_{j} q_{j}\right\rangle=\left[\frac{16}{3}\left|z_{i}\right|^{2}\left|z_{j}\right|^{2}-4\left(\left|z_{i}\right|^{2}+\left|z_{j}\right|^{2}\right)\right] \frac{\Lambda^{2}}{\mu},
$$

where the angular brackets denote the integration of Eq. (3) over $x$.

In order to average over the quantum coordinates $z_{i}$, we must select a suitable wave function for the baryon. Since direct analogues of the lowest-lying baryon octet do not exist in $\mathrm{QCD}_{2}$, we choose the $\Delta^{+}$state, which is the closest analogue of the proton in two dimensions. ${ }^{3}$ Its wave function is proportional to $z_{1}^{2} z_{2}$, and since we are interested in ratios of matrix elements, the normalization of the state factors out. Our convention is to normalize the $z$ integral (A5) to unity for $N=1, P=2$ when $N_{f}=N_{c}=3$, as is appropriate for the physical $\Delta^{+}$state.

We now discuss in more detail the internal symmetry properties of the bilinear $\overline{q_{i}} q_{j}$ and quadrilinear $\overline{q_{i}} q_{j} \overline{q_{k}} q_{\ell}$ operators. The isospin-one bilinear scalar densities are given in terms of the $u$ and $d$ fields by

$$
\begin{aligned}
|1,1\rangle & =(\bar{d} u), \\
|1,-1\rangle & =-(\bar{u} d), \\
|1,0\rangle & =\frac{(\bar{u} u)-(\bar{d} d)}{\sqrt{2}} .
\end{aligned}
$$

\footnotetext{
${ }^{3} \mathrm{We}$ note in passing that the $\Delta I=1 / 2$ rule is known experimentally to be valid for $\Omega^{-} \rightarrow \Xi \pi$ decays in $\mathrm{QCD}_{4}$ : the ratio of $\Delta I=3 / 2,1 / 2$ amplitudes $A_{3,1}$ is measured to be $A_{3} / A_{1}$ $=-0.063 \pm 0.014[14]$.
} 
The corresponding relations between the $I_{3}=0$ quadrilinear scalar densities of isospins $I=2,0$ and the quark fields are

$$
\begin{aligned}
T_{20} & =\left\langle\sqrt{\frac{2}{3}}\left[\frac{(\bar{u} u)-(\bar{d} d)}{\sqrt{2}}\right]^{2}-\frac{2}{\sqrt{6}}(\bar{u} d)(\bar{d} u)\right\rangle \\
& =\left\langle-\frac{1}{\sqrt{6}}\left\{2(\bar{u} d)(\bar{d} u)-[(\bar{u} u)-(\bar{d} d)]^{2}\right\}\right\rangle, \\
T_{00} & =\left\langle-\frac{1}{\sqrt{3}}\left\{2(\bar{u} d)(\bar{d} u)+\left[\frac{(\bar{u} u)-(\bar{d} d)}{\sqrt{2}}\right]^{2}\right\}\right\rangle \\
& =\left\langle-\frac{1}{2 \sqrt{3}}\left\{4(\bar{u} d)(\bar{d} u)+[(\bar{u} u)-(\bar{d} d)]^{2}\right\}\right\rangle .
\end{aligned}
$$

We then use Eq. (A2) to perform the $x$ integrations, obtaining

$$
\begin{array}{r}
T_{20} \propto\left[2\left|z_{1}\right|^{2}\left|z_{2}\right|^{2}-\left(\left|z_{1}\right|^{2}-\left|z_{2}\right|^{2}\right)^{2}\right] \frac{\Lambda^{2}}{\mu} \\
=\left[4\left|z_{1}\right|^{2}\left|z_{2}\right|^{2}-\left(\left|z_{1}\right|^{4}+\left|z_{2}\right|^{4}\right)\right] \frac{\Lambda^{2}}{\mu}, \\
T_{00} \propto \frac{4\left|z_{1}\right|^{2}\left|z_{2}\right|^{2}+\left(\left|z_{1}\right|^{2}-\left|z_{2}\right|^{2}\right)^{2}}{\sqrt{2}} \frac{\Lambda^{2}}{\mu} \\
=\frac{2\left|z_{1}\right|^{2}\left|z_{2}\right|^{2}+\left(\left|z_{1}\right|^{4}+\left|z_{2}\right|^{4}\right)}{\sqrt{2}} \frac{\Lambda^{2}}{\mu}
\end{array}
$$

for the different quadrilinear scalar densities with $I=2,0$, respectively.

The ratio of interest to us is

$$
\frac{\left\langle T_{20}\right\rangle}{\left\langle T_{00}\right\rangle}=\sqrt{2} \frac{2 R-1}{R+1},
$$

where the auxiliary ratio $R$ is defined by

$$
R \equiv 2 \frac{\left\langle\left|z_{1}\right|^{2}\left|z_{2}\right|^{2}\right\rangle}{\left\langle\left|z_{1}\right|^{4}+\left|z_{2}\right|^{4}\right\rangle} .
$$

Using the integrals (A5), we get $R=\frac{2}{3}$, which leads to the final result

$$
\frac{\left\langle T_{20}\right\rangle}{\left\langle T_{00}\right\rangle}=\sqrt{\frac{2}{5}} \approx 0.28
$$

for the ratio of quadrilinear scalar densities.

This ratio is not nearly as small as the corresponding ratio for current-current operators in $\mathrm{QCD}_{4}$ [14], even after allowing for Clebsch-Gordan coefficient factors of order unity and the calculable perturbative short-distance enhancement in the latter case [9]. However, before concluding anything about the significance of different nonperturbative effects in $\mathrm{QCD}_{2}$ and $\mathrm{QCD}_{4}$, we must analyze the technically more complicated case of bilinear products of Lorentz-vector currents in $\mathrm{QCD}_{2}$.

\section{FORMALISM FOR VECTOR CURRENTS AND COLLECTIVE-COORDINATE QUANTIZATION}

The extra complications in the calculations of hadronic matrix elements of Lorentz-vector currents and their bilinear products arise because one must take into account the time dependence of the hadronic wave function [2], an issue that has not been confronted in previous $\mathrm{QCD}_{2}$ calculations [4], including those in the previous section. These complications appear for the first time because the expressions for the vector currents involve time derivatives of the $z_{i}$, corresponding physically to the appearance of conjugate momenta.

To see how these arise, we first derive general expressions for the vector-current operators in terms of the classical coordinate $x$ and the quantum coordinates $z$. The usual bosonization rules yield, for the vector currents,

$$
\begin{aligned}
& J_{+}=\frac{i}{2 \pi} U^{\dagger} \partial_{+} U, \\
& J_{-}=\frac{i}{2 \pi} U \partial_{-} U^{\dagger},
\end{aligned}
$$

where $U$ is a nonlinear $\sigma$-model variable in the action of the bosonized representation of $\mathrm{QCD}_{2}$. The semiclassical quantization rules give the field $U$ in terms of a time-dependent "zero-mode" rotation $A(t)$ from the classical solution $U_{c}(x)$ :

$$
U=A(t) U_{c}(x) A^{\dagger}(t) .
$$

The last column of the zero-mode matrix $A$ is expressed in terms of the semiclassical variables $z_{i}$.

The light-front derivatives $\partial_{+,-} U$ may now be evaluated:

$$
\partial_{+,-} U=\dot{A}(t) U_{c} A^{\dagger}(t)+A(t) U_{c} \dot{A}^{\dagger}(t) \pm A(t) U_{c}^{\prime}(x) A^{\dagger}(t) .
$$

Using Eq. (14), we then find

$$
\begin{aligned}
U^{\dagger} \partial_{+} U= & A U_{c}^{\dagger}\left(A^{\dagger} \dot{A}\right) U_{c} A^{\dagger}+A \dot{A}^{\dagger}+A U_{c}^{\dagger} U_{c}^{\prime} A^{\dagger} \\
= & A\left[\left(U_{c}^{\dagger}-1\right)+1\right] A^{\dagger} \dot{A}\left[\left(U_{c}-1\right)+1\right] A^{\dagger} \\
& +A \dot{A}^{\dagger}+A U_{c}^{\dagger} U_{c}^{\prime} A^{\dagger} \\
= & A\left(U_{c}^{\dagger}-1\right) A^{\dagger} \dot{A}\left(U_{c}-1\right) A^{\dagger}+A\left(U_{c}^{\dagger}-1\right) A^{\dagger} \dot{A} A^{\dagger} \\
& +\dot{A}\left(U_{c}-1\right) A^{\dagger}+A U_{c}^{\dagger} U_{c}^{\prime} A^{\dagger}
\end{aligned}
$$

which yields the expression for the current $J_{+}$,

$$
\begin{aligned}
J_{+}= & \frac{i}{2 \pi}\left\{A\left(U_{c}^{\dagger}-1\right) A^{\dagger} \dot{A}\left(U_{c}-1\right) A^{\dagger}\right. \\
& \left.+\left[\dot{A}\left(U_{c}-1\right) A^{\dagger}-A\left(U_{c}^{\dagger}-1\right) \dot{A}^{\dagger}\right]+A\left(U_{c}^{\dagger} U_{c}^{\prime}\right) A^{\dagger}\right\},
\end{aligned}
$$

and an analogous expression may be derived for $J_{-}$.

Defining $\Phi \equiv \exp (i \beta \varphi)-1$, we may rewrite Eq. (16) in the form 


$$
\begin{aligned}
\left(J_{+}\right)_{k l}= & \frac{i}{2 \pi}\left[z_{k}|\Phi|^{2} z_{j}^{*} \dot{z}_{j} z_{l}^{*}+\dot{z}_{k} z_{l}^{*} \Phi^{*}\right. \\
& \left.-z_{k} \dot{z}_{l}^{*} \Phi-i \beta \varphi^{\prime}(x) z_{k} z_{l}^{*}\right]
\end{aligned}
$$

which may be simplified to

$$
\begin{aligned}
\left(J_{+}\right)_{k l}= & \frac{i}{2 \pi}\left[2(1-\cos \beta \varphi) z_{k}\left(z_{j}^{*} \dot{z}_{j}\right) z_{l}^{*}\right. \\
& \left.-i \beta \varphi^{\prime} z_{k} z_{l}^{*}+\dot{z}_{k} z_{l}^{*} \Phi-z_{k} \dot{z}_{l}^{*} \Phi\right] .
\end{aligned}
$$

The analogous calculation for the current $J_{-}$yields

$$
\begin{aligned}
\left(J_{-}\right)_{l k}= & \frac{i}{2 \pi}\left[2(1-\cos \beta \varphi) z_{l}\left(z_{j}^{*} \dot{z}_{j}\right) z_{k}^{*}\right. \\
& \left.-i \beta \varphi^{\prime} z_{l} z_{k}^{*}+\dot{z}_{l} z_{k}^{*} \Phi^{*}-z_{l} \dot{z}_{k}^{*} \Phi^{*}\right] .
\end{aligned}
$$

Equations (18) and (19) are the basis for our subsequent discussion of the matrix elements of the current-current operators.

It is convenient to rewrite Eqs. (19) and (18) in terms of the "momentum" variables

$$
\pi_{l} \equiv \frac{1}{2 M}\left[\dot{z}_{l}-z_{l}\left(z_{j}^{*} \dot{z}_{j}\right)\right]
$$

which are convenient for computational purposes, though they differ from the conjugate momenta resulting from the action by terms linear in $z$, as seen below in Eq. (26). We may rewrite the currents (18), (19) as follows in terms of the "momenta"' (20):

$$
\begin{aligned}
\left(J_{+}\right)_{k l}= & \left(\frac{i}{2 \pi}\right)\left\{(1-\cos \beta \varphi)(2 M)\left(z_{k} \pi_{l}^{*}-\pi_{k} z_{l}^{*}\right)\right. \\
& \left.-i(\sin \beta \varphi)(2 M)\left(\pi_{k} z_{l}^{*}+z_{k} \pi_{l}^{*}\right)-i \beta \varphi^{\prime} z_{k} z_{l}^{*}\right\}, \\
\left(J_{-}\right)_{l k}= & \left(\frac{i}{2 \pi}\right)\left\{(1-\cos \beta \varphi)(2 M)\left(z_{l} \pi_{k}^{*}-\pi_{l} z_{k}^{*}\right)\right. \\
& \left.+i(\sin \beta \varphi)(2 M)\left(\pi_{l} z_{k}^{*}+z_{l} \pi_{k}^{*}\right)-i \beta \varphi^{\prime} z_{l} z_{k}^{*}\right\}
\end{aligned}
$$

and are ready to address the computation of the matrix elements.

We now formulate the quantization of the quantummechanical soliton problem. In terms of the $z, \dot{z}$ variables, the bosonized effective action of $\mathrm{QCD}_{2}$ may be written as [2]

$$
S[z]=\frac{1}{2 M} \int d t(D z)^{\dagger}(D z)+\frac{i}{2} N_{c} \int d t\left[\dot{z}^{\dagger} z-z^{\dagger} \dot{z}\right]
$$

where $(D z)_{k}=\dot{z}_{k}-z_{k}\left(z_{l}^{*} \dot{z}_{l}\right)$ is the covariant derivative with respect to the auxiliary $\mathrm{U}(1)$ gauge field. The $z$ are subject to the constraints

$$
z^{*} z=1, \quad z^{*}(D z)=0
$$

where the first constraint follows from the $C P^{N-1}$ nature of the $z_{i}$-s and then the second follows from the definition of $D z$. The canonical momenta are given by

$$
p_{k}=\frac{\partial S}{\partial \dot{z}_{k}^{*}}=\frac{1}{2 M}\left[\dot{z}_{k}-z_{k}\left(z_{l}^{*} \dot{z}_{l}\right)\right]+\frac{i}{2} N_{c} z_{k}
$$

and there is a conjugate expression for $p_{k}^{*}$. It is convenient to rewrite the constraints (24) in the form

$$
\begin{aligned}
p_{k} & =\frac{1}{2 M}(D z)_{k}+\frac{i}{2} N_{c} z_{k}, \\
z^{*} p & =\frac{i}{2} N_{c} .
\end{aligned}
$$

In order to quantize the system of coordinates with constraints, we impose the commutation relations

$$
i\left[p_{k}, z_{l}^{*}\right]=\delta_{k l}-z_{k} z_{l}^{*}
$$

corresponding to the following commutation rules for the "momenta" $\pi_{i}$ :

$$
\left[z_{k}^{*}, \pi_{l}\right]=i\left(\delta_{k l}-z_{k}^{*} z_{l}\right)
$$

which maintain consistency with the constraints $z_{k} z_{k}^{*}=1$ and $z_{l}^{*} \pi_{l}=0$. The first of these follows from the unitarity condition on the matrix $A$, and the second follows from the first, combined with the definition of $\pi_{l}$, Eq. (20).

We now represent $i \pi_{l}$ by

$$
i \pi_{l}=\frac{\partial}{\partial z_{l}^{*}}-z_{l} z_{k}^{*} \frac{\partial}{z_{k}^{*}} .
$$

This representation incorporates the commutation rules Eq. (28) and the constraint $z_{l}^{*} \pi_{l}=0$, given that $z_{l}^{*} z_{l}=1$. Using Eq. (29), we find

$$
i \pi_{l} f\left(z, z^{*}\right)=i\left[\pi_{l}, f\left(z, z^{*}\right)\right]
$$

which will be used in the calculations of the following section.

\section{COMPUTATION OF THE MATRIX ELEMENTS FOR THE VECTOR CURRENT-CURRENT INTERACTION}

We now apply the formalism developed above to the calculation of the ratio of matrix elements in which we are interested. As in the scalar case, we calculate the following ratio of flavor combinations, where the Lorentz indices are left implicit:

$$
\frac{T_{20}}{T_{00}}=\sqrt{2} \frac{\left\langle\left[2(\bar{u} d)(\bar{d} u)-(\bar{u} u-\bar{d} d)^{2}\right]\right\rangle}{\left\langle\left[4(\bar{u} d)(\bar{d} u)+(\bar{u} u-\bar{d} d)^{2}\right]\right\rangle} .
$$

The numerator and the denominator in Eq. (31) involve similar products of Lorentz components of the vector currents,

$$
\begin{gathered}
\langle(\overline{u d})(\bar{d} u)\rangle \propto\left\langle\left(J_{+}\right)_{12}\left(J_{-}\right)_{21}\right\rangle+\left\langle\left(J_{-}\right)_{21}\left(J_{+}\right)_{12}\right\rangle, \\
\langle(\bar{u} u)(\bar{d} d)\rangle \propto\left\langle\left(J_{+}\right)_{11}\left(J_{-}\right)_{22}\right\rangle+\left\langle\left(J_{-}\right)_{11}\left(J_{-}\right)_{22}\right\rangle,
\end{gathered}
$$

and similarly for $\langle(\bar{u} u)(\bar{u} u)\rangle,\langle(\bar{d} d)(\bar{d} d)\rangle$. Recalling the expressions $(21),(22)$ for the components appearing in Eq. (32), 
we see that the evaluation of the matrix elements requires the following commutation relations equations derived from Eq. (28):

$$
\begin{aligned}
i\left[\pi_{1}^{*}, z_{1}^{2}\right] & =2 z_{1}\left(1-z_{1}^{*} z_{1}\right), \\
i\left[\pi_{1}^{*}, z_{2}\right] & =-z_{1}^{*} z_{2}, \\
i\left[\pi_{1}^{*}, z_{1}^{2} z_{2}\right] & =z_{1} z_{2}\left(2-3 z_{1}^{*} z_{1}\right), \\
i\left[\pi_{2}^{*}, z_{1}^{2} z_{2}\right] & =z_{1}^{2}\left(1-3 z_{2}^{*} z_{2}\right) .
\end{aligned}
$$

We will also need the results

$$
i\left(z_{1} \pi_{2}^{*}-\pi_{1} z_{2}^{*}\right) z_{1}^{2} z_{2}=z_{1}^{3}\left(1-2 z_{2}^{*} z_{2}\right)
$$

and

$$
i\left(\pi_{1} z_{2}^{*}+z_{1} \pi_{2}^{*}\right)^{2} z_{1}^{2} z_{2}=z_{1}^{3}\left(1-4 z_{2}^{*} z_{2}\right) .
$$

Using these relations, it is possible to rewrite $\langle(\overline{u d})$ $\times(\bar{d} u)\rangle$ in the form

$$
\left\langle\left(J_{+}\right)_{12}\left(J_{-}\right)_{21}+\text { H.c. }\right\rangle \propto A_{11}+A_{22}+A_{33}+\left(A_{31}+A_{13}\right),
$$

where the different terms appearing in Eq. (36) may be expressed as

$$
\begin{aligned}
A_{11}= & \frac{2(2 M)^{2}}{(2 \pi)^{2}}\left[\int d x(1-\cos \beta \varphi)^{2}\right] \int\left|z_{1}\right|^{6}(1 \\
& \left.-2\left|z_{2}\right|^{2}\right)^{2} \\
A_{22}= & -\frac{2(2 M)^{2}}{(2 \pi)^{2}}\left[\int d x(\sin \beta \varphi)^{2}\right] \int\left|z_{1}\right|^{6}(1 \\
& \left.-4\left|z_{2}\right|^{2}\right)^{2} \\
A_{33}= & \frac{2}{(2 \pi)^{2}}\left[\int d x\left(\beta \varphi^{\prime}\right)^{2}\right] \int\left|z_{1}\right|^{6}\left|z_{2}\right|^{4}, \\
A_{31}+A_{13}= & \frac{2(2 M)}{(2 \pi)^{2}}(2 \pi) \int\left|z_{1}\right|^{6}\left|z_{2}\right|^{2}\left(1-2\left|z_{2}\right|^{2}\right) .
\end{aligned}
$$

These different terms come from products and combinations of the three parts of Eqs. (21) and (22). It is easy to see that the only interference terms that survive the integral over $x$ are the $A_{13,31}$ terms exhibited in Eq. (38).

Performing the $x$ integrals over the classical configuration parametrized by $\varphi(x)$, and using formulas given in the Appendix, we obtain

$$
\begin{aligned}
A_{11} & =\frac{2 M^{2}}{\pi^{2}} \frac{1}{\mu}\left(\frac{16}{3}\right) \int\left|z_{1}\right|^{6}\left(1-2\left|z_{2}\right|^{2}\right)^{2}, \\
A_{22} & =-\frac{2 M^{2}}{\pi^{2}} \frac{1}{\mu}\left(\frac{8}{3}\right) \int\left|z_{1}\right|^{6}\left(1-4\left|z_{2}\right|^{2}\right)^{2}, \\
A_{33} & =\frac{1}{2 \pi^{2}}(8 \mu) \int\left|z_{1}\right|^{6}\left|z_{2}\right|^{4}, \\
\left(A_{31}+A_{13}\right) & =\frac{2 M}{\pi^{2}}(2 \pi) \int\left|z_{1}\right|^{6}\left|z_{2}\right|^{2}\left(1-2\left|z_{2}\right|^{2}\right) .
\end{aligned}
$$

Using the relation between the two mass scales $M$ and $\mu$ which is given in the Appendix, as well as the $z$ integrals collected there, we obtain finally the numerical value

$$
\langle(\bar{u} d)(\bar{d} u)\rangle=\frac{\mu}{7}\left[\frac{10}{27}+\left(\frac{2}{\pi}\right)^{2}\right]
$$

for the first terms in the numerator and denominator of Eq. (31).

Turning now to the remaining terms, we recall that

$$
\begin{aligned}
\left(J_{ \pm}\right)_{k k}= & \left(\frac{i}{2 \pi}\right)\left\{(1-\cos \beta \varphi)\left(z_{k} \pi_{k}^{*}-\pi_{k} z_{k}^{*}\right)(2 M)\right. \\
& \left.\mp i(\sin \beta \varphi)\left(\pi_{k} z_{k}^{*}+z_{k} \pi_{k}^{*}\right)(2 M)-i \beta \varphi^{\prime} z_{k} z_{k}^{*}\right\} \\
= & \left(\frac{i}{2 \pi}\right)\{(2 M)(1-\cos \beta \varphi) \\
& \times\left[z_{k} \pi_{k}^{*}-z_{k}^{*} \pi_{k}+i\left(1-z_{k} z_{k}^{*}\right)\right] \mp i(2 M)(\sin \beta \varphi) \\
& \left.\times\left[z_{k}^{*} \pi_{k}+z_{k} \pi_{k}^{*}-i\left(1-z_{k} z_{k}^{*}\right)\right]-i \beta \varphi^{\prime} z_{k} z_{k}^{*}\right\} .
\end{aligned}
$$

The commutation relations may then be used to evaluate

$$
\begin{aligned}
i\left(z_{1} \pi_{1}^{*}-z_{2} \pi_{2}^{*}\right) z_{1}^{2} z_{2} & =z_{1}^{2} z_{2}\left(2-3 z_{1}^{*} z_{1}\right)-z_{1}^{2} z_{2}\left(1-3 z_{2}^{*} z_{2}\right)^{2} \\
& =z_{1}^{2} z_{2}\left[1-3\left(\left|z_{1}\right|^{2}-\left|z_{2}\right|^{2}\right)\right] .
\end{aligned}
$$

Using this relation, we see that the action of the currents on the proton state gives

$$
\begin{aligned}
& {\left[\left(J_{ \pm}\right)_{11}-\left(J_{ \pm}\right)_{22}\right]|p\rangle} \\
& =\frac{1}{2 \pi}\left\{2 M(1-\cos \beta \varphi)\left[1-2\left(\left|z_{1}\right|^{2}-\left|z_{2}\right|^{2}\right)\right]\right. \\
& \quad \mp i(\sin \beta \varphi)(2 M)\left[1-4\left(\left|z_{1}\right|^{2}-\left|z_{2}\right|^{2}\right)\right] \\
& \left.\quad+\beta \varphi^{\prime}\left(\left|z_{1}\right|^{2}-\left|z_{2}\right|^{2}\right)\right\}\left(z_{1}^{2} z_{2}\right)|g\rangle
\end{aligned}
$$

which results in the amplitude

$$
\begin{aligned}
\mathcal{I}\left(x, z_{1}, z_{2}\right) \equiv & \left\langle p\left|\left[\left(J_{+}\right)_{11}-\left(J_{+}\right)_{22}\right]\left[\left(J_{-}\right)_{11}-\left(J_{-}\right)_{22}\right]\right| p\right\rangle \\
& +\left\langle[\cdots]_{-}[\cdots]_{+}\right\rangle \\
= & \frac{2}{(2 \pi)^{2}}\left|z_{1}\right|^{4}\left|z_{2}\right|^{2}\left\{(2 M)^{2}(1-\cos \beta \varphi)^{2}\right. \\
& \times\left[1-2\left(\left|z_{1}\right|^{2}-\left|z_{2}\right|^{2}\right)\right]^{2}-(2 M)^{2}(\sin \beta \varphi)^{2} \\
& \times\left[1-4\left(\left|z_{1}\right|^{2}-\left|z_{2}\right|^{2}\right)\right]^{2}+\left(4 \pi / N_{c}\right) \\
& \times\left(\varphi^{\prime}\right)^{2}\left(\left|z_{1}\right|^{2}-\left|z_{2}\right|^{2}\right)^{2}+4 M \beta \varphi^{\prime}(1-\cos \beta \varphi) \\
& \left.\times\left[1-2\left(\left|z_{1}\right|^{2}-\left|z_{2}\right|^{2}\right)\right]\left(\left|z_{1}\right|^{2}-\left|z_{2}\right|^{2}\right)\right\}
\end{aligned}
$$

for the second terms in the numerator and denominator of Eq. (31).

The next step is to perform the $x$ integration over the classical soliton configuration, which results in 


$$
\begin{aligned}
\int d x \mathcal{I}\left(x, z_{1}, z_{2}\right)= & \frac{1}{2 \pi^{2}}\left|z_{1}\right|^{4}\left|z_{2}\right|^{2}\left\{\frac{64 M^{2}}{3 \mu}\left[1-2\left(\left|z_{1}\right|^{2}-\left|z_{2}\right|^{2}\right)\right]^{2}-\frac{32 M^{2}}{3 \mu}\left[1-4\left(\left|z_{1}\right|^{2}-\left|z_{2}\right|^{2}\right)\right]^{2}\right. \\
& \left.+(8 \mu)\left(\left|z_{1}\right|^{2}-\left|z_{2}\right|^{2}\right)^{2}+(4 M)(2 \pi)\left[1-2\left(\left|z_{1}\right|^{2}-\left|z_{2}\right|^{2}\right)\right]\left(\left|z_{1}\right|^{2}-\left|z_{2}\right|^{2}\right)\right\} \\
= & \frac{\mu}{2 \pi^{2}}\left|z_{1}\right|^{4}\left|z_{2}\right|^{2}\left\{\frac{1}{6}\left(\frac{\pi}{N_{c}}\right)^{2}+N_{c}\left(\frac{\pi}{N_{c}}\right)^{2}\left(\left|z_{1}\right|^{2}-\left|z_{2}\right|^{2}\right)+\left[8-\left(\frac{\pi}{N_{c}}\right)^{2}\left(\frac{4}{3}+2 N_{c}\right)\right]\left(\left|z_{1}\right|^{2}-\left|z_{2}\right|^{2}\right)^{2}\right\} .
\end{aligned}
$$

Specializing to the choices $N_{f}=N_{c}=3$, we find

$$
\begin{aligned}
\int d x \mathcal{I}\left(x, z_{1}, z_{2}\right)= & \frac{\mu}{2 \pi^{2}}\left|z_{1}\right|^{4}\left|z_{2}\right|^{2}\left\{\frac{\pi^{2}}{54}+\frac{\pi^{2}}{3}\left(\left|z_{1}\right|^{2}-\left|z_{2}\right|^{2}\right)\right. \\
& \left.+\left(8-\frac{22 \pi^{2}}{27}\right)\left(\left|z_{1}\right|^{2}-\left|z_{2}\right|^{2}\right)^{2}\right\} .
\end{aligned}
$$

This may be evaluated using elementary integrals listed in the Appendix, yielding

$$
\left\langle(\bar{u} u-\bar{d} d)^{2}\right\rangle=\frac{\mu}{7}\left[-\frac{4}{27}+\left(\frac{2}{\pi}\right)^{2}\right] .
$$

Substituting this and Eq. (39) into Eq. (31), we obtain our final result

$$
\frac{T_{20}}{T_{00}}=0.54
$$

for the vector-current case.

\section{COMMENTS AND DISCUSSION}

We have shown in this paper that the matrix elements of $\Delta I=2$ four-fermion operators in $\mathrm{QCD}_{2}$ are not suppressed greatly by comparison with the corresponding $\Delta I=0$ operators. This conclusion holds true for both scalar-scalar and vector-vector four-fermion operators. The calculation of the latter case required the development of some formal machinery, including collective-coordinate quantization and the treatment of the time dependence of the quantized soliton, that had not been required for previous calculations in $\mathrm{QCD}_{2}$ [4].

What might be the significance of our results for the interpretation of the $\Delta I=1 / 2$ enhancement observed in $\mathrm{QCD}_{4}$ ? Clearly, $\mathrm{QCD}_{2}$ and $\mathrm{QCD}_{4}$ differ in many respects, particularly in the ultraviolet and the infrared. The asymptotic freedom of $\mathrm{QCD}_{4}$ yields logarithmic factors in the ultraviolet region, which are known to leading and nextto-leading order [9]. Evaluating these factors at any plausible renormalization scale, and multiplying them by the ratios of $T_{00} / T_{20}$ that we find does not give anything like the $\Delta I=1 / 2$ enhancement factor that is found in $\mathrm{QCD}_{4}$, even if one allows for Clebsch-Gordan factors of order unity.

What about the infrared features of $\mathrm{QCD}_{2}$ and $\mathrm{QCD}_{4}$ ? Both theories are known to have $\bar{q} q$ condensates, and calculations in $\mathrm{QCD}_{2}$ [4] of ratios of the different $\langle B|\bar{q} q| B\rangle$ are known to be in qualitative agreement with determinations based on the magnitude of the $\sigma$ term extracted from $\pi N$ scattering [6], and with four-dimensional Skyrme model calculations [5].

The feeble enhancement of the $\Delta I=0$ four-fermion operators is to be compared with the model-dependent result found in the four-dimensional Skyrme model [13]. Might the problem of the small enhancement that we find lie with the fact that the gluon condensate is relatively small in two dimensions, even vanishing in the large- $N_{c}$ limit and in the versions of the Skyrme model used in [13]? It has indeed been suggested that the large enhancement of the $\Delta I=1 / 2$ operator matrix elements in $\mathrm{QCD}_{4}$ might be due to gluon condensate effects [12]. Our results are certainly consistent with this idea, though by no means conclusive. How could this suggestion be tested more directly? One possibility might be to use a formulation of chiral soliton models in $\mathrm{QCD}_{4}$ in which gluon condensation effects are taken more into account.

Even though our present calculations are not conclusive for the resolution of the long-standing $\Delta I=1 / 2$ puzzle, we believe that they may help build up a conceptual framework in which it might be resolved. Some of the calculational formalism developed here may also be useful in future applications of $\mathrm{QCD}_{2}$.

\section{ACKNOWLEDGMENTS}

This research was supported by the Israel Science Foundation administered by the Israel Academy of Sciences and Humanities. The research of M.K. was supported in part by the Einstein Center at the Weizmann Institute and by a Grant from the G.I.F., the German-Israeli Foundation for Scientific Research and Development.

\section{APPENDIX}

In this appendix we collect some formulae useful at intermediate stages in the derivation of our results for the vector currents.

Some relevant $x$ integrals include

$$
\int_{-\infty}^{\infty}(\cos \beta \varphi-1) d x=-\frac{4}{\mu}
$$

and

$$
\int_{-\infty}^{\infty}(\cos \beta \varphi-1)^{2} d x=\frac{16}{3 \mu}
$$

used in the scalar-density calculation in Sec. II, and in addition 


$$
\begin{aligned}
& \int d x(\sin \beta \varphi)^{2}=\frac{8}{3 \mu}, \\
& \int d x\left(\beta \varphi^{\prime}\right)^{2}=8 \mu,
\end{aligned}
$$

used in the vector-current calculation in Sec. IV. For completeness, we also recall the relation between the two mass scales we have in the problem

$$
M=\frac{m}{2 \sqrt{2}}\left(\frac{\pi}{N_{c}}\right)^{3 / 2}=\left(\frac{\pi}{8 N_{c}}\right) \mu .
$$

Generic integrals over soliton wave functions are given by expressions of the general form

$$
\begin{aligned}
\left\langle\left|z_{i}\right|^{2 N}\left|z_{j}\right|^{2 P}\right\rangle= & \left\{\frac{\left(N+n_{i}\right) !\left(P+n_{j}\right) !}{\left[\left(N_{f}+N_{c}\right)+(N+P)-1\right] !}\right\} / \\
& \left\{\frac{n_{i} ! n_{j} !}{\left[\left(N_{f}+N_{c}\right)-1\right] !}\right\} \quad(i \neq j)
\end{aligned}
$$

which is the matrix element of $\left|z_{i}\right|^{2 N}\left|z_{j}\right|^{2 P}$ in a normalized state of the form $z_{1}^{n_{1}} \cdots z_{i}^{n_{i}} \cdots z_{N_{f}}^{n_{N_{f}}}$, with the constraint
$\Sigma_{i} n_{i}=N_{c}$. The latter constraint is a quantum consistency condition imposed by the Wess-Zumino term in the effective bosonic action, whose coefficient is $N_{c}$. To evaluate the matrix elements represented in Eq. (4) we use

$$
\begin{aligned}
\left\langle\left|z_{i}\right|^{2}\right\rangle & =\frac{n_{i}+1}{N_{c}+N_{f}}, \\
\left\langle\left|z_{i}\right|^{2}\left|z_{j}\right|^{2}\right\rangle & =\frac{\left(n_{i}+1\right)\left(n_{j}+1\right)}{\left(N_{c}+N_{f}\right)\left(N_{c}+N_{f}+1\right)} \quad(i \neq j), \\
\left\langle\left|z_{i}\right|^{4}\right\rangle & =\frac{\left(n_{i}+1\right)\left(n_{i}+2\right)}{\left(N_{c}+N_{f}\right)\left(N_{c}+N_{f}+1\right)},
\end{aligned}
$$

in particular. In the case $N_{f}=N_{c}=3$, and normalizing to 1 when $N=2$ and $P=1$, as is appropriate for the normalization of the $\Delta^{+}$state, we have the general formula

$$
\left\langle\left|z_{1}\right|^{2 N}\left|z_{2}\right|^{2 P}\right\rangle=\frac{5 !}{2} \frac{N ! P !}{(N+P+2) !}
$$

used in Secs. II and IV.
[1] G. 't Hooft, Nucl. Phys. B72, 461 (1974); B75, 461 (1974).

[2] Y. Frishman and J. Sonnenschein, Phys. Rep. 223, 309 (1993).

[3] J. Ellis, Y. Frishman, and M. Karliner, Phys. Lett. B 272, 333 (1991); J. Ellis, Y. Frishman, A. Hanany, and M. Karliner, Nucl. Phys. B382, 189 (1992).

[4] Y. Frishman and M. Karliner, Nucl. Phys. B344, 393 (1990).

[5] J. Donoghue and C. Nappi, Phys. Lett. 168B, 105 (1986).

[6] T. P. Cheng, Phys. Rev. D 13, 2161 (1976); J. Gasser et al., Phys. Lett. B 213, 85 (1988); M. E. Sainio, in Proceedings of the Sixth International Symposium on Meson-Nucleon Physics and Structure of the Nucleon, Blaubeuren, Germany, 1995, edited by D. Drechsel et al. (Tubingen University, Tubingen, 1995).

[7] J. Ellis and M. Karliner, presented at the International School of Nucleon Spin Structure, Erice, Italy, 1995 (unpublished); Report No. hep-ph-9601280 (unpublished).

[8] H.-Y. Cheng, Int. J. Mod. Phys. A 4, 495 (1989).
[9] G. Buchalla, A. J. Buras, and M. E. Lautenbacher, Rev. Mod. Phys. 68, 1125 (1996).

[10] H. P. Nilles and V. Visnjić-Triantafallou, Phys. Rev. D 19, 969 (1979).

[11] G. Adkins, C. Nappi, and E. Witten, Nucl. Phys. B228, 433 (1983); for $N_{f}=3$, E. Guadagnini, ibid. B236, 35 (1984); P. O. Mazur, M. A. Nowak, and M. Praszałowicz, Phys. Lett. 147B, 137 (1984).

[12] V. Antonelli, S. Bertolini, M. Fabbrichesi, and E. I. Lashin, Nucl. Phys. B469, 181 (1996).

[13] For a partial list of references, see J. Bijnens, H. Sonoda, and M. B. Wise, Phys. Lett. 140B, 421 (1984); M. Praszalowicz and J. Trampetic, ibid. 161B, 169 (1985); N. Toyota and K. Fujii, Prog. Theor. Phys. 75, 340 (1986); N. Toyota, ibid. 77, 688 (1987); K. Fujii, Y. Kondo, and S. Saito, Prog. Theor. Phys. Suppl. 102, 99 (1992).

[14] M. Bourquin et al., Nucl. Phys. B241, 1 (1984). 